\title{
Evolución histórica de la educación en derecho
}

\section{Historical evolution of law education}

Recibido 18/10/2021

\author{
Manuel Vicente Zometa Bolaños \\ Candidato a Doctor en Educación Superior de \\ la Facultad Multidisciplinaria de Occidente de la \\ Universidad de El Salvador \\ manuel.zometa2@ues.edu.sv \\ https://orcid.org/0000-0002-1825-4047
}

Aceptado 16/11/2021

\section{Referencia}

Zometa Bolaños, M. V. (2021). Evolución histórica de la educación en derecho. Revista Guatemalteca de Educación Superior, 5(1), 77-87. https://doi.org/10.46954/revistages.v5i1.76

\section{Resumen}

Palabras clave:

universidad, educacion en derecho, facultad de derecho.
OBJETIVO: el objetivo del presente texto es hacer una descripción breve de la evolución histórica de las facultades de derecho, desde la Universidad de Bolonia hasta la Universidad de El Salvador y pretende mostrar a través de un discurso diacrónico, aquellos aspectos que aún son visibles en la educación en derecho en las universidades de América Latina. MÉTODO: haciendo uso del método histórico descriptivo y lógico deductivo, se busca resaltar aquellas características fundamentales de la fundación y administración de las universidades como de la constitución y gobierno de las facultades de derecho, así mismo se hace una breve referencia a los contenidos estudiados y las cualidades de la formación en cada momento histórico. CONCLUSIÓN: finalmente se realiza una descripción de aquellos elementos que son visibles aún en la actualidad en las facultades de derecho de América Latina, tanto en lo administrativo, pedagógico y político. 


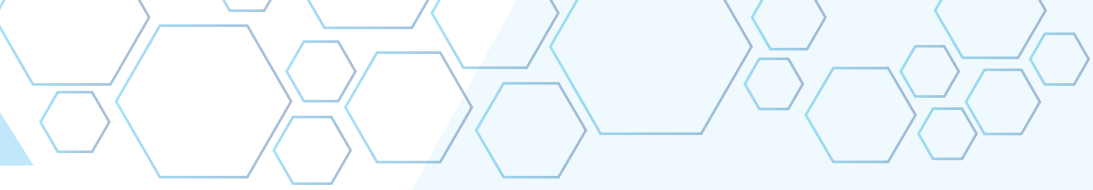

\section{Keywords:}

university, law education, law school.

\begin{abstract}
OBJECTIVE: the objective of this text is to make a brief description of the historical evolution of law schools from the University of Bolonia to the University of El Salvador. It aims to show through a diachronic discourse those aspects that are still visible in law education in Latin American universities. METHOD: using the historical descriptive and logical deductive method, the aim is to highlight those fundamental characteristics of the foundation and administration of the universities as well as the constitution and administration of the law schools, as well as a brief reference to the contents studied and the qualities of the education in each historical moment. CONCLUSION: finally, it describes those elements that are still visible today in law schools of Latin America, administratively, pedagogically and politically.
\end{abstract}

\section{Introducción}

Evolución histórica de la universidad y de la enseñanza del derecho, es una breve descripción del desarrollo de la <universitas> como institución aún antes de ser concebida como un centro de estudios, y busca hacer una sistematización diacrónica de cómo las primeras universidades y facultades de derecho se han venido consolidando hasta lo que son hoy en día; este ensayo se ha extraído de la tesis denominada: concepción pedagógica para la formación judicial en El Salvador, como trabajo de grado del Programa Interdisciplinario de Doctorado en Educación, con especialidad en Educación Superior, de la Facultad Multidisciplinaria de Occidente de la Universidad de El Salvador.

Mediante una revisión documental y la utilización de un método histórico descriptivo, se pretende resaltar aquellas características historicas de las facultades de derecho en las primeras universidades de América Latina, relacionando y describiendo las condiciones de su fundación, los contenidos esenciales de la formación a través de una linea discursiva basada en la ensenanza del derecho; asi mismo, se mencionan algunos de los alumnos y profesores destacados de la región; sin embargo, debido al alcance de este ensayo, no se realiza un abordaje sobre aspectos curriculares o programáticos de dichas universidades. 
El texto parte de la definición etimológica de universidad y cómo poco a poco su uso y contexto fue variando hasta llegar a lo que se conoce actualmente, luego se describe como la universidad y las facultades de derecho han evolucionado desde la primera universidad del mundo hasta llegar a la Universidad de El Salvador.

Finalmente, se hace una breve conclusión sobre aquellos aspectos que han sobrevivido o que estan aun vigentes e impactan en la educación en derecho en latinoamerica y en El Salvador.

\section{Contenido}

\section{Evolución histórica de la universidad y de la enseñanza del derecho}

Tomado a partir de la lengua romana, surge en la edad media el nombre <universitas> con el significado de "comunidad o corporación", utilizado para designar una institucion o asociación con miras a un fin determinado; fue hasta el siglo XII que este nombre fue reservado preferentemente a para denominar a los maestros y alumnos de las escuelas de París, convirtiendose así en la <universitas magistrorum et scholarium>, que fue la manera de definir a la personeria moral y jurídica de los centros de estudios superiores donde se impartía el <studium> generale, que se utilizaba para designar el conjunto de cursos estudiados (Posada, 2004, p. 29).

Así la palabra <universitas> adquirió la connotación de "gremio de los dedicados al oficio de enseñar y aprender", consecuentemente, surge la necesidad de definir a aquellos dedicados a enseñar y a aprender; en consecuencia, Isioro de Sevilla maestro de etimologías, propuso una expresion hermenéutica de la expresion <maior in statione> (el mayor en un lugar) basado en la palabra griega <steron>, así surge palabra <Magister> para designar a aquellos dedicados a enseñar dentro de la universitas; de igual manera, la palabra <Alumnus $>$ derivado del santo de alere que significa etimológicamente "alimentar o nutrir". 
Tanto los <magister> como los <alumnus> de disciplinas específicas, pactaron entre sí una serie de reglas sobre cómo organizar el proceso de enseñanza y aprendizaje, convirtiendose así en una pequena institución dentro de una corporación más grande, como lo era la <universitas>, surgiendo así las "facultades" (Facultas); para Gonzalo Posada (2004), fue esta organización la que colocó a la Universidad como el tercer poder del medioevo, justo despues de la iglesia y la monarquía.

A la cabeza de las <facultas>, se estableció a una persona encargada de dirigir y orientar las decisiones tomadas por el cuerpo de profesores al cual se denominó decano, mientras que a la cabeza de toda la < universitas> estaba el <amplissimus dominus rector $>$; quien era elegido según el modelo de cada universidad por los profesores titulares quienes poseían uno de los siguientes títulos: <magister, doctor, regens $>0$ <professor $>$.

La primera Universidad del mundo fue la Universidad de Bolonia fundada en 1088, dentro de sus primeras carreras se instituyó la de derecho, mediante el código de las siete partidas de Alfonso $X$ donde se establecieron los requisitos mínimos de la carrera y de los estudiantes de la <facultas $>$ de derecho (Universidad de Valencia, 2006), algunos de estos requisitos eran: ser hombre, sin defectos físicos ni enfermedades congénitas y saber leer y escribir latín.

En sus inicios, los alumnos más destacados de la carrera de Derecho, eran seleccionados para laborar como escribanos de justicia o como magistrados en las cortes de los reinados, teniendo a su cargo la administración de justicia, pero no la decisión final sobre los hechos a resolver, sino solamente la facultad para aconsejar al monarca y fue esta la figura que se instituyó no solo en Italia, sino también en España y Francia.

Desde la edad media, se venía pensando al derecho como ciencia, siendo los romanos quienes hicieron los aportes más significativos en relación a las leyes y sus instituciones, para Gonzalo Posada (2004), fueron los profesores de la Universidad de Bolonia quienes promovieron que las ciencias jurídicas se 
concibieran como una ciencia independiente de las ciencias sociales y la teología. Las primeras asignaturas que se impartieron sin embargo, fueron derecho canónico y derecho civil, esto con base en el trabajo de Bártolo de Sassoferrato denominado <utrumque ius> para el estudio del derecho canónico y el $<$ corpus iuris civilis $>$ para el estudio del derecho civil.

Ambos textos legales, son considerados como los más influyentes de la historia por ser la compilación mas importante de derecho canónico y romano respectivamente, ya que algunas de las máximas establecidas en este se encuentran aún vigentes en los sistemas de derecho neo-romano o continental.

Los primeros profesores de la Universidad de Bolonia fueron: Irineo, quien se especializaba en derecho romano; además aportó mucho a la enseñanza del derecho desde sus interpretaciones exegéticas y hermenéuticas del derecho romano, expresado mediante sus obras: <Summa codicis $>$ y $<$ Quaestiones de iuris subtilitatibus > y el segundo fue: Graciano, quien fuera un monge camaldulense que enseñaba derecho canónico y sus aportes fueron compilados en su obra <Concordia discordantium canonum>, conocida tambien como el "Decreto de Graciano" (Posada, 2004, p. 43).

Emulando el modelo de la Universidad de Bolonia y con algunos de sus alumnos egresados, el rey Alfonso IX de León promulgó las <Scholas Salamanticae> en 1218, que posteriormente se convirtió en la Universidad de Salamanca; lo que la convirtió en la primera universidad hispana; en 1254 por decreto del rey se nombró al primer maestro en leyes que impartia estudios sobre el <utrumque ius>, el <corpus iuris civilis> y se agregaron asignaturas en: decretales, lógica, gramática latina y española (Universidad de Salamanca, 2021).

En 1255 El rey Alejandro IV, publica las bulas pontificias que reconocen los grados académicos otorgados por la Universidad de Salamanca y se le otorga su propio sello; este hito fue muy importante para la historia de las universidades de Latinoamérica, ya que este modelo de fundación de universidad 
mediante cedula real y posterior bula pontificia, fue la manera en que se instituyeron casi todas las universides americanas, lo que significó una expansión sin precedentes (Universidad de Salamanca, 2021).

En Latinoamerica, los primeros abogados provenían de España y aplicaban las leyes derivadas de la Constitución Política de la Monarquía Española de 1812, tambien conocida como la "Constitucion de Cádiz" o "la Pepa", que dentro de su articulado regía sobre el territorio español "de ambos hemisferios", el europeo y el americano. (Constitución política de la Monarquia Española, 1812).

Estos abogados españoles en Latinoamérica, no solamente eran los designados para la función judicial en el virreinato, sino que también tenían control sobre el acceso al estudio y ejercicio de la profesion jurídica. Según Germán Silva (2006), los estudios jurídicos solamente podían cursarse en España y solo podían acceder los Españoles o sus desendientes legítimos, lo cual se comprobaba mediante lo que se denominó "los juicios de sangre" (García, 2006).

La primera Universidad Latinoamericana creada bajo el modelo de salamanca fué la Universidad Nacional Mayor de San Marcos, establecida en el entonces virreinato del Perú, su oficialización mediante Cédula Real y Real Provisión emitida por el rey Carlos I de España y V del Sacro Imperio Romano Germánico, le da el carácter de la primera universidad de Latinoamérica, a pesar que en 1538 se fundó la Universidad Santo Tomas de Aquino, mediante bula <in apostulatus culmine> del Papa Paulo III, se estima que jurídicamente no tuvo efecto por no haber seguido el proceso de formación salamanqués. (Valcárcel, 2010).

De hecho, la cédula real de la Universidad de San Marcos alude a directamente al modelo de la Universidad de Salamanca, y niega la validez jurídica de la bula que creó la Universidad de Santo Tomas de Aquino, la referida cédula establece lo siguiente: 
DON CARLOS por la Divina Clemencia, Emperador siempre Augusto, Rey de Alemania. Doña Juana su Madre, y el mismo Don Carlos por la gracia de Dios, Reyes de Castilla, de León, de Aragón, de las dos Cicilias, de Jerusalén, de Navarra, de Granada, de Toledo, de Valencia, de Galicia, de Mallorcas, de Sevilla, de Cerdeña, de Córdova, de Córcega, de Murcia, de Jaén, de los Algarves, de Algezira, de Gibraltar, de las Islas Canarias, de las Indias, Islas, é Tierra Firme del Mar Océano, Condes de Flandes, y de Tirol, \&. Por quanto Fray Thomas de Sant- Martín de la Orden de Santo Domingo, Provincial de la dicha Orden en las Provincias del Perú, nos ha hecho relación, que en la Ciudad de los Reyes de las dichas Provincias está hecho, y fundado un Monasterio de su Orden en el cual ay buen aparejo para se hazer Estudio General el qual sería muy provechoso en aquella tierra: porque los hijos de los Vecinos de ella, serían doctrinados, y enseñados, y cobrarían abilidad, é nos suplicó fuessemos servidos de tener por bien, que en el dicho Monasterio oviesse el dicho Estudio General con los privilegios, franquezas, y livertades, que ha, y tiene el Estudio y Universidad de la Ciudad de Salamanca, ó como la nuestra merced fuesse, y nos por el bien y ennoblecimiento de aquella tierra, hemoslo havido por bien por ende por la presente tenemos por bien, y es nuestra merced, y voluntad, que en el dicho Monasterio de Santo Domingo de la Ciudad de los Reyes, por el tiempo que nuestra voluntad fuere, entre tanto se dá orden como esté en otra parte donde más convenga en la dicha Ciudad pueda haver, y aya el dicho Estudio General, el qual tenga, y goze de todos los privilegios, franquezas y excepciones, que tiene, y goza el Estudio de la dicha Ciudad de Salamanca, con tanto q. en lo que toca á la jurisdicción, se guarde, y esté como ahora está, y que la Universidad de el dicho Estudio no execute jurisdicción alguna, é con los que allí se graduaren no gozen la livertad, que el Estudio de Salamanca tiene de no pechar los allí graduados. Y mandamos al nuestro Presidente, y Oydores de la nuestra Audiencia Real de las dichas Provincias del Perú, y otras qualesquier nuestras Justicias de ellas, y de las otras Islas, y Provincias de 
nuestras Indias, que guarden, y cumplan esta nuestra Carta, y lo en ella contenido, y contra el thenor, y forma de ella, ni de lo en ella contenido no vayan, ni passen, ni consientan ir, ni passar en tiempo alguno, por alguna manera. Dada en la Villa de Valladolid a doce dias del mes de Mayo de mil quinientos cinquenta y un años (Valcárcel, 2010).

Luego de la Universidad de San Marcos, en el virreinato de nueva españa en 1551, se funda por real cédula del emperador Carlos V la Real y Pontificia Universidad de México, aún que comenzó a funcionar hasta el año de 1553 siempre bajo el modelo de la <Schola Salamanticae>, las primeras facultades fueron las de Teología, Derecho y Medicina; a diferencia de otras universidades, en la Universidad de Mexico se estudiaban en todas las carreras los siete artes medievales según la agrupación tradicional: el <trivium>, (gramática, retorica y lógica) el <quadrivium> (aritmética, geometría, música y astronomía) y al ser el latín la lengua en la que se estudiaba, (por la denominacion de pontificia) tambien era necesario cursar el estudio de la lengua con un <Magister Scholarum> quien era el designado del papa en este hemisferio (Puente, 2000, pp. 29-49).

Posteriormente, en el año 1676 fue establecida en la Capitanía General de Guatemala en el mismo virreinato de Nueva España, por mandato del Rey Carlos II de España la primera universidad de Centro América: la Universidad de San Carlos, para lo cual fue designado como primer rector, el ex fiscal de la Real Audiencia Española y Juez Superintendente del virreinato Juan Bautista Urquiola y Elorriaga; quien fuera abogado, graduado en la Universidad de Salamanca y quien instituyó los primeros estudios superiores en derecho en la actual Guatemala (Rodriguez, 2020).

En 1687 el papa inocencio XI otorgó a la Universidad de San Carlos el título de "pontificia", lo que significó que sus graduados poseían el mismo nivel académico y su título, la misma validez que los de las universidades europeas. Las primeras asignaturas establecidas por la Real Cédula de Fundación de la universidad fueron Teología Escolástica, Teología Moral, Cánones, Leyes, Medicina y dos de lenguas extranjeras. 
El primer estudiante salvadoreño de la Universidad de San Carlos fue el Doctor José Matias Delgado de León, graduado como doctor en cánones y principal líder de la independencia de El Salvador; si bien no fue estudiante de derecho, estableció durante su período como diputado provincial de Guatemala, las leyes que permitieron que se fundaran universidades en cada uno de los Estados miembros de la entonces República Federal Centroamericana desde 1824, gracias a la influencia del pensamiento ilustrado.

El 2 de febrero de 1841, la República de El Salvador fue declarada Estado independiente a consecuencia de la extinción de la República Federal Centroamericana y el 16 de febrero de 1841, por decreto legislativo de la asamblea constituyente se funda la Universidad de El Salvador (Duran, 2009), en sus inicios fundó la facultad de medicina y la facultad de derecho con el nombre de " Facultad de jurisprudencia y ciencias sociales", las primeras cátedras de derecho que se intituyeron, fueron las de derecho civil y derecho canónico en el año de 1843, las cuales fueron asignadas al Dr. Francisco Dueñas ex presidente de El Salvador y al ex diputado de la extinta Federación Centroamericana, Pbro y Dr. Isidro Menéndez quienes fueron juristas graduados de la Universidad de San Carlos en Guatemala (Granadino, 2021).

\section{Conclusión}

Desde Roma hasta la actualidad, el estudio del derecho ha sido considerado como fundamental, no solo como objeto de estudio en sí mismo, sino que tambien por su cercania, dependencia e influencia de las estructuras del poder de cada época. Desde la época medieval, las clases dominantes como la iglesia y la monarquía fomentaban la formació de juristas, para poder legitimarse a través de las leyes y de la aplicación de ellas.

Durante el periodo de la ilustración, con el surgimiento de la idea de la separación entre la iglesia y el Estado, la educación en derecho tambien fue paulatinamente separandose de la influencia de la iglesia, pero continuaba siendo un instrumento útil para la legitimación del poder, ahora no solo por la función judicial sino tambien por la función legislativa propia de los Estados republicanos y democráticos. 
En la Actualidad, la mayoria de facultades de derecho en América Latina y en Europa, continuan formando abogados bajo un modelo de formación tradicional, con la misma afinidad y congruencia con los modelos de gobierno de cada Estado; sin embargo, la vinculación entre las facultades de derecho y los Gobiernos, ya es tan directa ni utilitaria como habia venido siendo al menos hasta el siglo XX.

\section{Referencias}

Constitución política de la Monarquia Española. (1812). https://www.minex.gob.gt/ADMINPORTAL/Data/ DOC/20100930181309440Consti1812Cadiz. Monarqu\%C3\%ADa.pdf

Duran, M. A. (2009). Historia de la Universidad de El Salvador. El Universitario, XIII(6), 9. http://www. editorialuniversitaria.ues.edu.sv/sites/default/files/ pdf/Revista\%20La\%20U\%2020\%20corregida\%20 completa\%20\%281\%29.pdf

García, G. S. (2006). Prospectivas sobre la educación jurídica. En R. P. Perdomo, \& J. Rodríguez Torres, La formación jurídica en America Latina (1ra ed., págs. 12 - 29). Universidad Externado de Colombia ISBN 958-710-058-1.

Granadino, J. O. (20 de octubre de 2021). Origen y desarrollo de la Facultad de Jurisprudencia y Ciencias Sociales y la enseñanza del derecho en El Salvador. Obtenido de jurisprudencia.ues.edu.sv: jurisprudencia.ues.edu.sv/ historia.html

Posada, G. S. (2004). La humanitas como universitas en el medioevo. En G. V. Guillén, Filosofía, pedagogía y enseñanza de la filosofía (1a edición ed., págs. 42 - 44). Editorial pedagógica nacional. https://revistas.udea.edu.co/ index.php/unip/article/view/12166

Puente, L. P. (2000). Universidad de doctores, Mexico en el siglo XVII. UNAM-CESU.https://novohispana.historicas.unam. mx/index.php/ehn/article/view/3563/3118 
Rodriguez, L. (28 de enero de 2020). Universidad San

Carlos de Guatemala . Obtenido de guatemala.com: https://aprende.guatemala.com/centros-educativos/ universidades/universidad-de-san-carlos-de-guatemala/

Universidad de Valencia. (2006). Obtenido de Historia de los estudios de derecho: uv.es/ceshu/estudios/estudios.pdf

Universidad de Salamanca. (20 de octubre de 2021). Historia de la Universidad de Salamanca Obtenido de usal.es: https://www.usal.es/historia

Valcárcel, C. D. (2010). Fundación de la Uniersidad de Lima (12 de Mayo de 1551). Obtenido de https://sisbib.unmsm. edu.pe/bibvirtual/libros/historia/San_marcos/Fund_Uni_ Lima.htm

\section{Sobre el autor}

Manuel Vicente Zometa Bolaños, docente e investigador de la Universidad de El Salvador, Profesor de evaluación de los aprendizajes en la masetria en la profesionalización de la docencia superior en la escuela de posgrados de la Facultad Multidisciplinaira de Occidente y profesor de filosofia del derecho en el departamento de derecho de la misma facultad.

\section{Conflicto de intereses}

Declaro no tener ningún conflicto de interes de ningún tipo en el proceso de publicación del presente ensayo.

\section{Declaración de consentimiento informado}

El estudio se realizó con fines educativos, respetando el Código de ética y buenas prácticas editoriales de publicación.

\section{Derechos de uso}

Copyright@ 2021 por Manuel Vicente Zometa Bolaños

Este texto está protegido por la Licencia Creative Commons Atribución 4.0 Internacional.

Usted es libre para compartir, copiar y redistribuir el material en cualquier medio o formato y adaptar el documento, remezclar, transformar y crear a partir del material para cualquier propósito, incluso comercialmente, siempre que cumpla la condición de atribución: usted debe reconocer el crédito de una obra de manera adecuada, proporcionar un enlace a la licencia, e indicar si se han realizado cambios. Puede hacerlo en cualquier forma razonable, pero no de forma tal que sugiera que tiene el apoyo del licenciante o lo recibe por el uso que hace. 